\title{
Basic science: (November 2008)
}

1. Alcaraz J, Xu R, Mori $H$, Nelson CM, Mroue R, Spencer VA, Brownfield D, Radisky DC, Bustamante $\mathrm{C}$, Bissell MJ. Laminin and biomimetic extracellular elasticity enhance functional differentiation in mammary epithelia. EMBO J 2008; 27: 2829-2838.

2. Behmoaram E, Bijian K, Jie S, Xu YJ, Darnel A, Bismar TA, Alaoui-Jamali MA. Focal adhesion kinase-related proline-rich tyrosine kinase 2 and focal adhesion kinase are co-overexpressed in early-stage and invasive ErbB-2-positive breast cancer and cooperate for breast cancer cell tumorigenesis and invasiveness. Am J Pathol 2008; 173: 1540-1550.

3. Brinton LA, Richesson D, Leitzmann MF, Gierach GL, Schatzkin A, Mouw T, Hollenbeck AR, Lacey JV. Menopausal hormone therapy and breast cancer risk in the NIH-AARP Diet and Health Study Cohort. Cancer Epidemiol Biomarkers Prev 2008; 17: 3150-3160.

4. Chlebowski RT, Johnson KC, Kooperberg C, Pettinger M, Wactawski-Wende J, Rohan T, Rossouw J, Lane D, O'Sullivan MJ, Yasmeen S, Hiatt RA, Shikany JM, Vitolins M, Khandekar J, Hubbell FA. Calcium plus vitamin D supplementation and the risk of breast cancer. $J$ Natl Cancer Inst 2008; 100: 1581-1591.

5. Harbeck N, Nimmrich I, Hartmann A, Ross JS, Cufer T, Gruetzmann R, Kristiansen G, Paradiso A, Hartmann O, Margossian A, Martens J, Schwope I, Lukas A, Mueller V, Milde-Langosch K, Nahrig J, Foekens J, Maier S, Schmitt M, Lesche R. Multicenter study using paraffin-embedded tumor tissue testing PITX2 DNA methylation as a marker for outcome prediction in tamoxifen-treated, nodenegative breast cancer patients. J Clin Oncol 2008; 26: 5036-5042.

6. Helczynska K, Larsson AM, Mengelbier LH, Bridges $E$, Fredlund $E$, Borgquist $S$, Landberg G, Pahlman S, Jirstrom K. Hypoxia-Inducible Factor-2 $\alpha$ correlates to distant recurrence and poor outcome in invasive breast cancer. Cancer Res 2008; 68: 9212-9220.

First published online 17/07/09 BCO/848/2009/JW
7. Johansson H, Gandini S, Guerrieri-Gonzaga A, lodice S, Ruscica M, Bonanni B, Gulisano M, Magni P, Formelli F, Decensi A. Effect of fenretinide and low-dose tamoxifen on insulin sensitivity in premenopausal women at high risk for breast cancer. Cancer Res 2008; 68: 9512-9518.

8. Kim H, Chung H, Kim HJ, Lee JY, Oh MY, Kim Y, Kong $\mathrm{G}$. Id-1 regulates $\mathrm{BCl}-2$ and $\mathrm{Bax}$ expression through p53 and NF- $\mathrm{B}$ in MCF-7 breast cancer cells. Breast Cancer Res Treat 2008; 112: 287-296.

9. Kruschinski A, Moosmann A, Poschke I, Norell $H$, Chmielewski M, Seliger B, Kiessling R, Blankenstein T, Abken $\mathrm{H}$, Charo J. Engineering antigen-specific primary human NK cells against HER-2 positive carcinomas. Proc Natl Acad Sci USA 2008; 105: 17481-17486.

10. Lee E, Ma H, McKean-Cowdin R, Van Den Berg D, Bernstein L, Henderson BE, Ursin G. Effect of reproductive factors and oral contraceptives on breast cancer risk in BRCA1/2 mutation carriers and noncarriers: results from a populationbased study. Cancer Epidemiol Biomarkers Prev 2008; 17: 3170-3178.

11. Mathes RW, Malone KE, Daling JR, Davis S, Lucas SM, Porter PL, Li Cl. Migraine in postmenopausal women and the risk of invasive breast cancer. Cancer Epidemiol Biomarkers Prev 2008; 17: 3116-3122.

12. Nikolsky Y, Sviridov E, Yao J, Dosymbekov D, Ustyansky V, Kaznacheev V, Dezso Z, Mulvey L, Macconaill LE, Winckler W, Serebryiskaya T, Nikolskaya T, Polyak K. Genome-wide functional synergy between amplified and mutated genes in human breast cancer. Cancer Res 2008; 68: 9532-9540.

13. Pankratz VS, Hartmann LC, Degnim AC, Vierkant RA, Ghosh K, Vachon CM, Frost MH, Maloney SD, Reynolds C, Boughey JC. Assessment of the accuracy of the Gail Model in women with atypical hyperplasia. J Clin Oncol 2008; 26: 5374-5379.

14. Phillips GDL, Li GM, Dugger DL, Crocker LM, Parsons KL, Mai E, Blattler WA, Lambert JM, Chari RVJ, Lutz RJ, Wong WLT, Jacobson FS, Koeppen H, Schwall RH, Kenkare-Mitra SR, Spencer SD, Sliwkowski MX. Targeting HER2positive breast cancer with Trastuzumab-DM1, 
an antibody-cytotoxic drug conjugate. Cancer Res 2008; 68: 9280-9290.

15. Plo I, Laulier C, Gauthier L, Lebrun F, Calvo F, Lopez BS. AKT1 inhibits homologous recombination by inducing cytoplasmic retention of $B R C A 1$ and RAD51. Cancer Res 2008; 68: 9404-9412.

16. Provenzano PP, Inman DR, Eliceiri KW, Beggs $\mathrm{HE}$, Keely PJ. Mammary epithelial-specific disruption of focal adhesion kinase retards tumor formation and metastasis in a transgenic mouse model of human breast cancer. Am J Pathol 2008; 173: 1551-1565.

17. Rottenberg S, Jaspers JE, Kersbergen A, van der Burg E, Nygren AOH, Zander SAL, Derksen PWB, de Bruin M, Zevenhoven J, Lau A, Boulter R, Cranston A, O'Connor MJ, Martin NMB, Borst P, Jonkers J. High sensitivity of BRCA1-deficient mammary tumors to the PARP inhibitor AZD2281 alone and in combination with platinum drugs. Proc Natl Acad Sci USA 2008; 105: 17079-17084.

18. Saunus JM, French JD, Edwards SL, Beveridge DJ, Hatchell EC, Wagner SA, Stein SR, Davidson A, Simpson KJ, Francis GD, Leedman PJ, Brown MA. Posttranscriptional regulation of the breast cancer susceptibility gene $B R C A 1$ by the RNA binding protein HuR. Cancer Res 2008; 68: 9469-9478.

19. Schwabedissen H, Tirona RG, Yip CS, Ho RH, Kim RB. Interplay between the nuclear receptor Pregnane $\mathrm{X}$ receptor and the uptake transporter organic anion transporter Polypeptide $1 \mathrm{~A} 2$ selectively enhances estrogen effects in breast cancer. Cancer Res 2008; 68: 9338-9347.

20. Sieri S, Krogh V, Ferrari P, Berrino F, Pala V, Thiebaut ACM, Tjonneland A, Olsen A, Overvad K, Jakobsen MU, Clavel-Chapelon F, Chajes V, Boutron-Ruault MC, Kaaks R, Linseisen J, Boeing H, Nothlings U, Trichopoulou A, Naska A, Lagiou P, Panico S, Palli D, Vineis P, Tumino R, Lund E, Kumle M, Skeie G, Gonzalez CA, Ardanaz E, Amiano P, Tormo MJ, Martinez-Garcia C, Quiros JR, Berglund G, Gullberg B, Hallmans G, Lenner P, Bueno-de-Mesquita HB, van Duijnhoven FJB, Peeters PHM, van Gils CH, Key TJ, Crowe FL, Bingham S, Khaw KT, Rinaldi S, Slimani N, Jenab M, Norat T, Riboli E. Dietary fat and breast cancer risk in the European prospective investigation into cancer and nutrition. Am J Clin Nutr 2008; 88: 1304-1312.

21. Sonestedt E, Borgquist S, Ericson U, Gullberg B, Olsson H, Adlercreutz H, Landberg G, Wirfalt E. Enterolactone is differently associated with estrogen receptor $\beta$-negative and -positive breast cancer in a Swedish nested casecontrol study. Cancer Epidemiol Biomarkers Prev 2008; 17: 3241-3251.
22. Su G, Blaine SA, Qiao DH, Friedl A. Membrane type 1 matrix metalloproteinase-mediated stromal Syndecan-1 shedding stimulates breast carcinoma cell proliferation. Cancer Res 2008; 68: 9558-9565.

23. Van der Auwera I, Van Laere SJ, Van den Bosch SM, Van den Eynden GG, Trinh BX, van Dam PA, Colpaert CG, van Engeland M, Van Marck EA, Vermeulen PB, Dirix LY. Aberrant methylation of the Adenomatous Polyposis Coli (APC) gene promoter is associated with the inflammatory breast cancer phenotype. Br J Cancer 2008; 99: 1735-1742.

24. Van Laere S, Beissbarth T, Van der Auwera I, Van den Eynden G, Trinh XB, Elst $\mathrm{H}$, Van Hummelen $P$, van Dam P, Van Marck E, Vermeulen P, Dirix L. Relapse-free survival in breast cancer patients is associated with a gene expression signature characteristic for inflammatory breast cancer. Clin Cancer Res 2008; 14: 7452-7460.

25. Walker LC, Waddell N, Ten Haaf A, Grimmond S, Spurdle $A B$. Use of expression data and the CGEMS genome-wide breast cancer association study to identify genes that may modify risk in BRCA1/2 mutation carriers. Breast Cancer Res Treat 2008; 112: 229-236.

26. Wayne S, Neuhouser ML, Ulrich CM, Koprowski C, Wiggins C, Baumgartner KB, Bernstein L, Baumgartner RN, Gilliland F, McTiernan A, Ballard-Barbash R. Association between alcohol intake and serum sex hormones and peptides differs by tamoxifen use in breast cancer survivors. Cancer Epidemiol Biomarkers Prev 2008; 17: 3224-3232.

27. Wolf I, Levanon-Cohen S, Bose S, Ligumsky H, Sredni B, Kanety H, Kuro-o M, Karlan B, Kaufman B, Koeffler HP, Rubinek T. Klotho: a tumor suppressor and a modulator of the IGF-1 and FGF pathways in human breast cancer. Oncogene 2008; 27: 7094-7105.

28. Zhang HJ, Liu G, Dziubinski M, Yang ZQ, Ethier SP, Wu GJ. Comprehensive analysis of oncogenic effects of PIK3CA mutations in human mammary epithelial cells. Breast Cancer Res Treat 2008; 112: 217-227.

29. Zhao JJ, Lin JH, Yang $H$, Kong $W$, He LL, Ma X, Coppola D, Cheng JQ. MicroRNA-221/222 negatively regulates estrogen receptor $\alpha$ and is associated with tamoxifen resistance in breast cancer. J Biol Chem 2008; 283: 31079-31086.

Prepared by

$R$ Sutherland

Cancer Research Program Garvan Institute of Medical Research Darlinghurst, NSW, Australia 